\title{
Ueber die concentrischen Körper der Thymus.
}

\author{
Von
}

Dr. B. A fanassiew aus St. Petersburg.

(Anatomisches Institut zu Strassburg.)

Hierzu Taf. I.

Gelegentlich einer Untersuchung über die entwickelungsgeschichtlichen und vergleichend anatomischen Verhältnisse der Thymus fielen mir einige Befunde auf, welche geeignet erscheinen, auf die Bildungsweise der bekannten, so charakteristischen concentrischen Körper der Drüse einiges Licht zu werfen und uns zugleich den Weg zeigen, der zur regulären Rückbildung des Organs führt.

Die von Ecker sogenannten concentrischen Thymuskörper, deren erste Keuntniss wir Hassall, The microscopical anatomy of the human body in health and disease, London, 1846, verdanken, Henle (Handbuch der rat. Pathol.) bezeichnete sie deshalb mit dem Namen der "Hassall'schen Körperchen" -, sind von den verschiedenen Autoren, welche mit ihrer Entstehungsgeschichte sich befasst haben, in verschiedener Weise gedeutet worden, doch hat bis jetzt, meiner Meinung nach, Niemand das Richtige getroffen.

Eine Reihe von älteren Angaben, wie die von Simon, Henle, Ecker (für einen Theil der Körper wenigstens) bringen die Entstehung der fraglichen Gebilde mit einer Fettmetamorphose der Drüsenzellen zusammen. Ha s a ll selbst hält sie für "Mutterzellen “ und beschreibt sie als Gebilde, die zahlreiche Kerne enthalten, deren jeder von einer oder mehreren concentrischen Lamellen umgeben sei«. Er vergleicht sie den Knorpelzellen in den Zwischenwirbelknorpeln und den Zellen von gewissen Algenspecies der Gattung Microcystis zugehörig. Auch $G \ddot{u} n s b u r g$, s. dessen Zeitschrift für klinische Medicin Bd. VIII. 1857, p. 456 ff., scheint die concentrischen Körper als Mutterzellen aufzufassen. Vir chow, Arch. für pathol. Anat. III. Band, p. 222, stellt sie in einer kurzen Bemerkung hinsichtlich ihrer Entstehung mit den concentrischen Cancroidkörpern zusammen. Letztere wieder liess bekanntlich Virchow damals durch Umlagerung von Krebszellen um Zellen mit sogenannten Bruträumen sich heranbilden. 
Kölliker (Handbuch der Gewebelehre 5. Aufl), dem Jendrassik (Wiener anad. Sitzungsb. 1856) zustimmt, spricht sich dahin aus, dass in den meisten Fällen die fraglichen Bildungen nicht durch Umwandlungen und concentrische Agglomeration aus den Zellen der Drüse, wie bereits Ecker (Artikel: "Blutgefässdrüsen«, Wagner's Handwörterbuch der Physiologie) es darstellte, sondern durch allmähliche Umlagerung eines nicht zelligen Materiales um dieselben entständen, also den Prostataconcrementen verwandt wären ; vielleicht seien sie durch Umwandlungen von Eiweissstoffen der Drüse entstanden. Uebrigens gibt Kölliker zu, dass in anderen Fällen diese Körper aus concentrisch in einander geschachtelten platten Zellen beständen. Für die zellige Structur spricht sich auch His, Zeitschrift f. wissensch. Zool. Bd. X p. 348 und Frey, Lehrbuch der Histologie und Histochemie 5. Aufl. 1877 aus. W. Krause gibt an, Handbuch der allgem. Anatomie I, p. 359, dass die Zerlegung der Körperchen in platte polygonale kernhaltige Epithelzellen leicht durch Behandlung mit Müller'scher Flüssigkeit gelinge.

Die eingehendsten Beschreibungen der concentrischen Thymuskörper liefern wohl Berlin, Archiv für die Holländischen Beiträge zur Natur- und Heilkunde Bd. I p. 230, und Paulitzky. Ersterer hat keine näheren Angaben über die Entwickelung dieser Gebilde; es findet sich indessen bei ihm der nicht näher erläuterte Satz: (p. 233) "Sie (die concentrischen Körper) liegen meistens in der Nähe von Gefässen oder sind geradezu durch dieselben begrenzt». Für Involutionsgebilde möchte er sie nicht gelten lassen; gibt jedoch an, dass sie bei jüngern Föten ( 5 monatl. menschlichen) fehlen. Er lässt in ihnen endogen Kerne und Zellen gebildet werden, die nachher austreten und frei werden sollen. Paulitzky (Disquisitiones de stratis glandulae thymi corpusculis, Halis, 1863) stellt die etwas wunderliche Ansicht auf, dass die geschichteten Körper aus eingeschachtelten "Epithelzellen" entständen; letztere wiederum gingen aus den "Bindegewebszellen" des Thymusgerüstes hervor.

His l. c. und Bruch (Zeitschr. f. rat. Med. I. Ser. IX. Bd.) leiten die Körperchen von abgeplatteten Drüsenzellen ab, wobei Letzterer eine regressive Metamorphose derselben betont. Friedleben (die Physiol. der Thymusdrüse, Frankfurt a. M. 1858) hingegen lässt sie auf dem Wege regressiver Metamorphose aus ganzen sog. "Drüsenblasen" der Thymus entstehen. Bezüglich des Verhaltens zu den Gefässen finden wir auch bei His eine bemerkenswerthe 
Angabe: "Die concentrischen Körper finden sich in der Regel in Verbindung mit kleineren Gefässen, oft umgeben sie dieselben vollständig, oft sitzen sie an den Theilungswinkeln auf, und man findet daher, dass sie beim Auspinseln feiner Thymusschnitte nicht weggespült werden, sondern im Zusammenhang mit den Gefässen bleiben. " Paulitzky bestätigt dieses Verhalten. Friedleben erwähnt Verdickungen der Wandung bei den Thymusarterien mit nachfolgender Obliteration, Erweiterung und varicöser Entartung der Venen während der Involution des Organes, ohne jedoch nähere histologische Angaben über diese Veränderungen zu machen.

Wir finden also noch eine ganze Anzahl differenter Ansichten über die concentrischen Körper, von denen übrigens keine eine befriedigende Auskunft darüber gibt, wie diese Körper sich bilden.

Wie mir nun zahlreiche Präparate aus menschlichen, Kaninchenund Kalbsdrüsen ergeben haben, stammen di e concentrischen Körper von den Gefässendothelien ab. Letztere, namentlich die der venösen Gefässe und der Capillaren, vergrössern sich zuvörderst - Fig. 2 Taf. I mag als Beleg dafür dienen - und vermehren sich, wie ich vertreten zu können glaube, durch Theilung und füllen dann bald die Gefässlumina aus. Gleichzeitig mit dieser Wucherung der Endothelien geht vielfach eine Abschnürung der betreffenden Gefässröhren in kleinere Abschnitte von verschiedener Grösse einher. Man trifft an sorgfältig hergestellten Präparaten (Härtung der frischen Drüsen in Ammon. monochromic., dann Auswaschen in aq. destill. und Alkohol, Auspinselung und Färbung in Haematoxylin und ammoniakalischer Eosinlösung) alle nur wünschbaren Uebergangsstufen in hinreichend dünnen Schnitten: Gefässröhren mit vergrösserten Endothelzellen, ferner Röhren, deren Lumina von solchen Zellen ganz verstopft erscheinen, endlich solche, bei denen die Endothelien concentrisch geschichtet sind und das Lumen ganz oder zum Theil ausfüllen. Den sichersten Beweis für die Richtigkeit der von mir hier vorgebrachten Auffassung sehe ich indessen in Bildern, wie sie in Fig. 1 und 2 Taf. I wiedergegeben sind. Hier zeigen sich - und Eosinfärbung leistet dabei besonders gute Dienste - im Innern der concentrischen Körper noch wohl erhaltene Blutkörperchen. Es lässt der letzterwähnte Befund wohl kaum daran zweifeln, dass die hier beschriebenen Bilder richtig gedeutet seien. Es sei hier ausdrücklich hervorgehoben, dass 
Jendrassik, l. c. p. 95, nicht selten kleine Blutextravasate im Parenchym der Thymus fand und bei dieser Gelegenheit sagt: "Auch schien es mir hier und da, dass veränderte Blutzellen in manchen concentrischen Körpern selbst enthalten waren.“ Er spricht jedoch an keiner Stelle von Gefässveränderungen oder einer etwaigen Bildung der concentrischen Körper aus den Gefässen, obgleich er sonst diesen letzteren eine sehr detaillirte Beschreibung widmet.

Selbstverständlich ist auch nicht versäumt worden, diese Befunde durch Injectionspräparate zu controlliren. Ich habe hierbei besonders von der menschlichen'. Thymus, öfter Schnitte erhalten, in denen man die Injectionsmasse bis zu concentrischen Körpern, die das betreffende Gefässrohr verstopften, verfolgen konnte; ausserdem mehreremale solche anscheinend frei liegende Körper, welche mit der Injectionsmasse gefärbt waren.

Mit Rücksicht auf die Angaben früherer Autoren habe ich auch mein Augenmerk darauf gerichtet, ob etwa von den übrigen zelligen Elementen der Thymus, also von den Thymuszellen oder den Zellen des reticulums aus, eine Entstehung der zelligen Elemente der concentrischen Körper nachzuweisen wäre - allein ich habe nichts dergleichen constatiren können. Dass eine Abschnürung der so veränderten Gefässe vorliegen müsse, darf wohl daraus geschlossen werden, dass man seltener Körper von länglicher oder verästelter Gestalt antrifft, wie es doch wohl der Fall sein müsste, wenn eine Abschnürung nicht Statt fände. Uebrigens sieht man auch sehr ausgeprägt als solche erkennbare Längsansichten von Gefässen, welche mit gewucherten Endothelzellen vollgepfropft sind, und in denen bereits eine concentrische Lagerung der Endothelien begonnen hat, vergl. besonders Fig. 1 Taf. 1.

Weiterhin ist zu erwähnen, dass die Gefässwand gewöhnlich bei dem ganzen Vorgange verdickt erscheint und wohl die kapselartigen Bildungen erzeugen dürfte, die man um viele concentrische Körper findet.

In andern Fällen wiederum scheinen die gewucherten Endothelzellen eine regressive Metamorphose (Zerfall in körnige, fetthaltige Massen) einzugehen. S. Fig. 1.

Die hier vertretene Auffassung der Bildung der concentrisehen Körper harmonirt sehr wohl mit der verschiedentlich (Bruch, Ecker, Paulitzky) geäusserten Angabe, dass die concentrischen Körper am zahlreichsten während der Involutions-Periode vorkämen. 
Von Berlin, His und Friedleben ist dem widersprochen worden. Meiner und Prof. Waldeyer's Erfahrung nach fehlen während der ersten Entwickelungszeit der Thymus die concentrischen Körperchen gänzlich und kommen während der Involutionszeit am häufigsten vor. Sie fehlen allerdings nicht zu einer Zeit, in der die Thymus noch wächst, aber es können ja sehr gut einzelne Abschnitte der Drüse einer regressiven Metamorphose unterliegen, während andere neu gebildet werden, bez. weiter wachsen.

In der mir zugängigen Literatur habe ich eine Angabe über die Bildung von concentrischen Körpern aus dem Blutgefässendothel der Thymus nicht gefunden; überhaupt dürften nicht viele Beobachtungen bezüglich der Entstehung concentrischer Körper aus Blutbez. Lymphgefässendothelien vorliegen, wenn mir von der bekannten Köster'schen Monographie: "Die Entwickelung der Carcinome und Sarcome, Würzburg 1869 , in der die Carcinome und auch deren concentrische Körperchen von den Lymphgefässendothelien abgeleitet werden, hier absehen.

Offenbar sind die herichteten Thatsachen in mehr als einer Beziehung von Interesse. Zunächst dürfte wohl in dem auf diese Weise erfolgenden massenhaften Untergange von Gefässen der Thymus der Weg zur regulären physiologischen Rückbildung des Organs gegeben sein. Wir erfahren freilich durch die mitgetheilten Befunde noch Nichts über die näheren Ursachen dieser Gefässveränderung; immerhin wären wir aber einen Schritt weiter zur Erklärung dieses merkwürdigen Factums gekommen. Die concentrischen Körper treten damit aus der Reihe der einfach histologischen Curiositäten heraus. Weiterhin ist darauf aufmerksam zu machen, dass hier eine Localität vorliegt, in der mir unzweifelhaft die Entstehung von concentrisch geschichteten Zellenballen aus end othelialen Elementen gegeben scheint; für die Frage nach der Verwerthung der concentrischen Körper bei der anatomischen Diagnose von Krebsgeschwülsten hat diese Thatsache sicherlich ihren Werth. (Vgl. das oben citirte Werk Köster's.)

Endlich dürfte darauf hinzuweisen sein, dass vielleicht auch noch an andern Körperstellen Gefässe auf diese Art obliteriren, oder dass wenigstens concentrische Endothelialkörper in ihnen entstehen. Man kann die Möglichkeit wohl zugeben, dass solche Körper durch den andrängenden Blutstrom später wieder flott gemacht werden, und würden so vielleicht die Angaben Hassall's vom Vor- 
kommen concentrisch geschichteter Körper im Blute eine bessere Erklärung fanden als bisher.

\section{Erklärung der Figuren.}

Fig. 1. Combinirt aus mehreren dünnen Durchschnitten einer Thymus. a, a, a : eine kleine Vene der Länge nach getroffen, Wandung stark verdickt, bedeutende Wucherung des Endothels mit einzelnen kleinen concentrischen Körperchen. b. Querschnitt einer Capillare mit sehr grossen Endothelzellen. c. Concentrisches Körperchen wit einem rothon Blutkörperchen. d und f. Querschnitte kleiner Venen mit gewuchertem und zerfallenem Endothel; in d noch eine Anzahl rother Blutkörper. e. Querschnitt einer Capillare; in einer Endothelzelle ein kleiner Pigmentfleck (Hämatin).

Fig. 2. a, b, c. Querschnitte und Längsschnitte kleiner Venen mit verdickten Wänden und stark entwickeltem Endothel; von b geht ein kleiner Seitenast ab, dessen Lumen von Zellen ganz ausgefüllt ist. d. eine Capillare, welche in eine kleine Vene einmündet; in beiden das Endothel vermehrt und seine Zellen vergrössert. e, e, e, e, grosse dunkelkörnige Zellen, welche sich oft in grosser Menge in der Umgebung der Thymusgefässe finden (Plasmazellen, Waldeyer).

\section{Die Anlage der Keimblätter bei den Diplopoden (Chilognathen).}

Ein Beitrag zur Entwicklungsgeschichte der Myriopoden.

Von

Ant. Stecker in Prag.

Hierzu Taf. II.

Die ersten Entwicklungsvorgänge, die Furchung und Blätterbildung sind bei den Myriopoden noch so wenig bekannt, dass ich hier in Kürze die von mir in dieser Beziehung gemachten Beobachtungen mittheilen will. Da ich aber nur die Diplopoden untersucht habe, und keine günstige Gelegenheil fand, auch an den, 


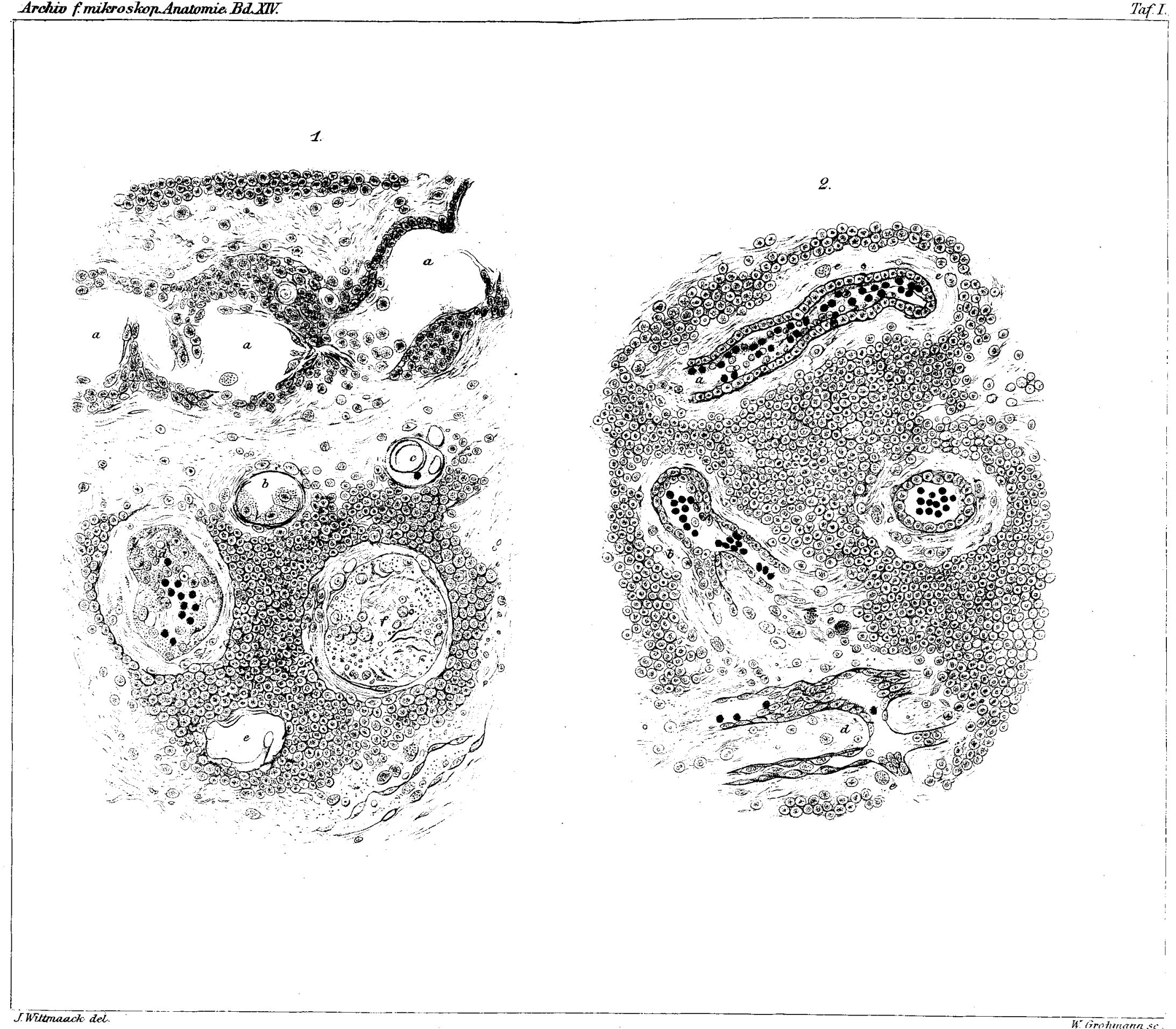

